\title{
Frecuencia de infección por chlamydia trachomatis en el endocervix y el endometrio de un grupo de mujeres infertiles comparadas con un grupo fértil.
}

\author{
María Helena Vásquez*, Luis Fernando Barrera**, Fabio Sánchez***, Federico Díaz****
}

\begin{abstract}
RESUMEN: Se compararon 60 pacientes con problemas de infertilidad con 120 mujeres multíparas; se investigó la presencia de Chlamydia trachomatis tanto en el endometrio como en el endocervix, utilizando la inmunofluorescencia directa (MICROTRAK). Los controles tenían significativamente mayor edad, más años de vida sexual y mayor número de abortos. Con relación a la presencia de C.trachomatis se encontró mayor positividad en el grupo control tanto en el endocervix (33\% vs. $12 \%$ no significante), como en el endometrio (38\% vs. $17 \%$ ), como también mayor positividad simultánea en ambos sitios anatómicos, sin embargo sólo se encontró diferencia significativa (p 0.005$)$ en cuanto a la muestra endometrial en favor del grupo control. Se llama la atención hacia la alta prevalencia de infección por C. trachomatis en la-población de mujeres fértiles.
\end{abstract}

PALABRAS CLAVES: Chlamydia trachomatis, endocervix, endometrio infertil, mujeres fertiles.

SUMMARY: Sixty women with fertility problems were compared with a control group of 120 multiparous ones; the presence of Chlamydia trachomatis was determined, both in endocervical and in endometrial specimens, by means of a direct inmunofluorescence test (MICROTRAK). The following parameters were significantly highler ( $\left.\begin{array}{ll}p & 0.001\end{array}\right)$ in the control group: age, years of sexual activity, numbers of live children and of abortions. Regarding Chlamydia trachomatis a higher positivity rate was found in the multiparous group both in the endocervix (33\% vs. 12\%, nonsignificant) and in the endometrium ( $38 \%$ vs. $17 \%, p \quad 0.005)$

Simultaneous positivity in both specimens was also higher in the control group but the difference was non-significant. We call the attention to the high frecuency of $C$. trachomatis infection in a fertile population.

Primer premio modalidad Residente Concurso Nacional efectuado por la Asociación Antioqueña de Obstetricia y Ginecología. 1990.

KEY WORDS: Chlamydia trachomatis, endocervix, endometrium sterility, fertility women.

\section{Introducción}

El conocimiento de la patogénesis de la infección del tracto genital femenino por los serotipos oculogenitales de Chlamydia trachomatis está en una fase de rápida evolución. Se considera en la actualidad que en muchos aspectos la Chlamydia se comporta como la Neisseria gonorrhoeae., esto es, que la transmisión se realiza fundamentalmente por contacto sexual con un hombre portador de este microorganismo y que produce en la mujer infección a nivel del epitelio cilíndrico en el endocervix, la uretra y el recto; posteriormente se puede producir infección del tracto genital superior como resultado de diseminación canalicular del microorganismo, dando origen, en algunas mujeres, a secuelas graves tales como embarazo ectópico, enfermedad inflamatoria pélvica e infertilidad; también puede producir infección perinatal como resultado de la inoculación del microorganismo durante el parto, la cual suele manifestarse en el recién nacido como neumonía y conjuntivitis de inclusión.

\footnotetext{
* Profesor Titular Dpto. Microbiología y Parasitología, Facultad de Medicina, U. de A.

** Médico Residente Dpto. Ginecología y Obstetricia, Facultad de Medicina, U. de A.

*** Profesor Titular Dpto. Ginecología y Obstetricia, Facultad de Medicina, U. de A.

**** Profesor Titular Dpto. Microbiología y Parasitología, Facultad de Medicina, U. de A., Director Revista IATREIA.
}

Se acepta que en los países desarrollados los serotipos oculogenitales de Chlamydia trachomatis son los agentes más prevalentes de enfermedades de transmisión sexual (1, 2). La prevalencia de infección por Chlamydia trachomatis en el endocervix es muy diferente, de acuerdo a los resultados de distintas investigaciones y poblaciones estudiadas, es así como en la población general oscila entre el 3\% y el $5 \%$ (1); sin embargo, en poblaciones seleccionadas la prevalencia es mayor; por ejemplo: varía del $12 \%$ al $31 \%$ en clínicas de enfermedades sexualmente transmisibles (3-12); del $14,7 \%$ al $39 \%$ en adolescentes sexualmente activas (1316), del 27\% (15) al $47 \%$ (16) en las adolescentes embarazadas; del $5,5 \%$ al $22,5 \%$ entre las usuarias de instituciones de planificación familiar $(17,18)$; del $40 \%$ en pacientes con enfermedad inflamatoria pélvica $(16,19)$ y en mujeres embarazadas que solicitan aborto terapéutico la prevalencia oscila del $5 \%$ al $17,9 \%(20,21)$.

En otros trabajos se ha buscado la Chlamydia en sitios diferentes del endocervix con los siguientes resultados: $24 \%$ de aislamiento a partir del endometrio en pacientes con salpingitis aguda (22) y $8 \%$ de positividad en las trompas de Falopio en pacientes con enfermedad inflamatoria pélvica (EIPA) (19).

Mardh y col. (23) fueron los primeros en sugerir que la. Chlamydia trachomatis es un agente etiológico de enfermedad inflamatoria pélvica aguda (EIPA); es así como se ais- 
laron en el endocervix de $19(36 \%)$ sobre 53 pacientes con EIPA, y de las trompas de $6(30 \%)$ de 20 pacientes con dicha enfermedad.

Como consecuencia de esta participación en la etiología de la EIPA se ha atribuido a la Chlamydia trachomatis un posible papel en el origen de la infertilidad. Entre las pacientes con EIPA en Europa Occidental casi un 30\% alberga este microorganismo en el endocervix, en contraste con un $10 \%-20 \%$ de positivas para Neisseria gonorrhoeae en el mismo sitio. (24) Se ha informado sobre la aparición de endometritis clamidiásica en asociación con EIPA, fenómeno que apoya aún más evidencias de la diseminación luminal de esta bacteria en esta entidad (25).

La etiología del aborto habitual se logra determinar en un porcentaje que oscila entre $56 \%$ a $63 \%(28,29)$; y el resto de pacientes se clasifica en el grupo de causa desconocida. No se ha establecido aún el papel de la Chlamydia en el aborto habitual. Schachter (26) pudo aislarla en tenidos de abortos en 4 de.22 (18\% especímenes, sin embargo Cracea y col. (27) no lograron demostrar la presencia de Chlamydia en cultivos tomados de mujeres con historia de abortos a repetición.

En nuestro medio, no hay estudios que investiguen en forma sistemática la presencia de la Chlamydia trachomatis en mujeres infértiles, es por esto que nos propusimos invetigar su presencia en este grupo de pacientes; además relacionarlo con un grupo control, con el interés de evaluar el verdadero papel de este microorganismo en la infertilidad.

\section{Materiales y metodos}

Se realizó la investigación utilizando el modelo de casos y controles.

En el grupo de casos se incluyeron 60 pacientes de clase socioeconómica baja que consultaron por inferilidad al grupo de ginecología Endocrina y Reproducción Humana del Hospital Universitario San Vicente de Paúl (H.U.S.V.P.-Medellín), con una edad comprendida entre 17 y 40 años, y que no habían recibido tratamiento con tetraciclinas en los seis meses previos. Este grupo fue dividido en tres subgrupos iguales de 20 pacientes cada uno, así: a) infertilidad primaria, definida como la paciente que no ha tenido ningún embarazo luego de un año de vida sexual y sin utilizar método anticonceptivo alguno; b) infertilidad secundaria, definida como la paciente que ha tenido uno o más embarazos o abortos y que no se ha embarazado luego de un año de vida sexual, sin empleo de métodos anticonceptivos; c) abortadora habitual, definida como la paciente que ha tenido 3 abortos sucesivos o 4 alternos.

El grupo control lo constituyeron 120 pacientes multíparas, con dos o más embarazos, con edades comprendidas entre 17 y 40 años, sexualmente activas, que no planificaban con dispositivo intrauterino (DIU) ni con anticonceptivos orales y que no habían recibido tetraciclinas en los 6 meses previos a la toma de las muestras; todas estas pacientes consultaron a una clínica de planificación familiar (Profamilia-Medellín) con el fin de iniciar un método de planificación. Este grupo pertenecía a la clase socioeconómica media.

Todas las pacientes fueron seleccionadas ál azar; a todas se les diligenció un formulario que incluía los siguientes Jatos: edad, estado civil, años de vida sexual activa, número de compañeros sexuales, número de relaciones sexuales por mes y total de gestaciones, aborto y paridad.

A cada paciente se le tomaron muestras de endocervix y de cavidad uterina, para la búsqueda de infección por Chlamydia trachomatis utilizando la técnica de Inmunofluorescencia directa (ID).

Para la toma de la muestra endocervical se colocó un espéculo vaginal estéril no lubricado, se limpió el orificio cervical por medio de un escobillón estéril con el fin de remover el exceso de moco; se introdujo otro escobillón estéril en el canal endocervical, se frotó y rotó durante diez segundos con el fin de obtener la mayor cantidad de células de descamación; la muestra se extendió sobre una lámina portaobjetos, dentro de un pozo excavado de $8 \mathrm{~mm}$. de diámetro, extendiéndola en toda el área; las láminas fueron secadas y fijadas con $0,5 \mathrm{ml}$. de metanol, que se dejó evaporar totalmente a temperatura ambiente; las placas fijadas fueron conservadas a $-20^{\circ} \mathrm{C}$ hasta su lectura.

Para la toma de la muestra endometrial, se fabricó un dispositivo por uno de los autores que consistió en adaptar el aplicador del dispositivo intrauterino ( $\mathrm{T}$ de cobre), el cual consta de una camisa (tubo de plástico) en cuyo interior va un aplicador de alginato de calcio esterilizado con óxido de etileno. (Fig. No. 1). El dispositivo se introdujo hasta el fondo de la cavidad uterina y con el aplicador se frotó el endometrio con el fin de obtener un material rico en células, luego se retiró el aplicador a través de la camisa para evitar la contaminación con flora endocervical y se procedió a realizar los extendidos y la fijación con la técnica ya descrita.

Técnica de Inmunofluorescencia [Micro-trak]. Se utilizaron anticuerpos monoclonales contra la membrana proteica externa que es común a los quince serotipos de Chlamydia trachomatis, conjugados con isotiocianato de fluoresceína, según la técnica standar (30).

Las muestras fueron leídas en un microscopio de fluorescencia Leitz, utilizando aumentos de 40x y 100x. Se leyó como positiva aquélla muestra en donde se observaron diez o más cuerpos elementales por preparación. En todos los casos se leyeron simultáneamente controles positivos y negativos.

Se utilizó un microcomputador APC IV marca NEC, y el programa estadístico Statgraphics versión 2.6 y tablas de $2 \times 2$ para el análisis estadístico.

Para efectos del análisis estadístico, el grupo de casos se tomó en conjunto, ya que cuando se analizaron cada uno de los subgrupos por separado no se encontró ninguna diferencia significativa.

\section{Resultados}

Cuando se analizan las dos poblaciones con relación a las variables estudiadas se puede apreciar que existe una diferencia significativa $(p<0,001)$ en cuanto a la edad: en el grupo control el promedio es $33,19 \pm 0,42$ años y en el grupo de casos 29,38 $\pm 0,70$ años; también hay diferencia significativa en cuanto a los años de vida sexual activa $(\mathrm{p}<$ 0.001 ), con promedio de $13,34 \pm 0,50$ para el control y de $7,78 \pm 0,69$ para los casos.

Por las características de las poblaciones estudiadas existe diferencia significativa $(\mathrm{p}<0,001)$ con relación al número de gestaciones, con $\mathrm{x} 4,32 \pm 0,18$ para el grupo control $\mathrm{y} \mathrm{x}$ 
$2,30 \pm 0,34$ para los casos, diferencia que es mayor cuando se analiza el número de hijos vivos con un $x 3,37 \pm 0,15$ para el control contra $x 0,55 \pm 0,11$ en los casos. Igual acontece en cuanto al número de abortos con x 1,38 $\pm 0,23$ para los casos y x $0,50 \pm 0,08$ para los controles. (Tabla No. 1).

Tabla 1

MEDIA Y DESVIACION ESTANDAR DE VARIABLES QUE PRESENTARON SIGNIFICANCIA ESTADISTICA

\begin{tabular}{|llll|}
\hline Variable & $\begin{array}{l}\text { Controles } \\
\mathbf{n}=120\end{array}$ & $\begin{array}{l}\text { Casos } \\
\mathrm{n}=60\end{array}$ & $\begin{array}{l}\text { Nivel de } \\
\text { significancia }\end{array}$ \\
\hline Edad & $33.19 \pm 0.42$ & $29.38 \pm 0.70$ & $\mathrm{P}<0.001$ \\
Años Vida & & & $\mathrm{P}<0.001$ \\
Sexual & $13.34 \pm 0.50$ & $7.78 \pm 0.69$ & $\mathrm{P}<0.001$ \\
No. Gestaciones & $4.32 \pm 0.18$ & $2.30 \pm 0.34$ & $\mathrm{P}<0.001$ \\
No. Hijos vivos & $3.37 \pm 0.15$ & $0.55 \pm 0.11$ & $\mathrm{P}<0.0001$ \\
No. Abortos & $0.50 \pm 0.08$ & $1.38 \pm 0.23$ & $\mathrm{P}$ \\
\hline
\end{tabular}

El $84 \%$ de las pacientes en ambos grupos poblacionales son casadas. No existe diferencia significativa entre ambos grupos con relación al número de compañeros sexuales, con un promedio de uno, ni con respecto al número de relaciones por mes, cuyo promedio fue siete en ambos grupos.

Con relación a la positividad para Chlamydia trachomatis en los dos sitios anatómicos estudiados, se aprecia, contrario a lo esperado, una mayor prevalencia en el grupo control tanto en el endocervix, como en el endometrio y simultáneamente en ambos sitios; al hacer el análisis estadístico, sin embargo, sólo se encuentra diferencia significativa $(\mathrm{p}<$ 0,005 ) en cuanto a la muestra endometrial, en el grupo control.

Al analizar la proporción de positividad de la infección endocervical por Chlamydia trachomatis en un cuadro de $2 \times 2$ se encuentra que es mayor en los controles $(32.5 \%)$ que en los casos $(20 \%)$, diferencia que sin embargo no es significativa, $(\mathrm{p}=0.11)$; la razón de disparidad $(\mathrm{Rd})$ es de 0,52 (Tabla No. 2).

En cuanto a la infección endometrial, la proporción de positividad es mayor en el grupo control (38\%) que en el grupo de casos (17\%), diferencia que es significativa, $(\mathrm{p}=0.005)$; la razón de disparidad $(\mathrm{Rd})$ es de 0,32 .

Al considerar la positividad de la Chlamydia trachomatis simultáneamente en los dos sitios anatómicos la proporción de positividad es mayor para el grupo control (20\%) que para el grupo de casos (12\%), diferencia que no es significativa, $(\mathrm{p} \pm 0.23)$; la razón de disparidad ( $\mathrm{Rd})$ es 0,53 .

\section{Discusión}

En estudios realizados en ratones y monos, se ha evidenciado, que posterior a la infección de las trompas de Falopio por $\mathrm{C}$. trachomatis, se observa un severo daño tisular, caracterizado por una marcada desciliación, inmovilidad permanente de la cilias y necrosis de las células secretoras, pudiéndose también producir piosalpinx y obstrucción tubárica; estos efectos sobre el epitelio se explican por la localización intracelular del patógeno y por la peculiaridad de su ciclo biológico (31).

Por la capacidad que tiene la Chlamydia de producir lesiones inflamatorias en el endocervix, el endometrio y el

Tabla No. 2

\section{FRECUENCIA DE INFECCION ENDOCERVICAL} POR CHLAMYDIA TRACHOMATIS

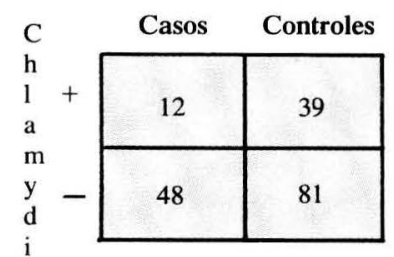
FRECUENCIA DE INFECCION ENDOMETRIAL
POR CHLAMYDIA TRACHOMATIS

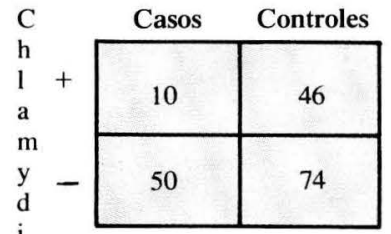

Proporción de positividad de casos $10 / 10+50=17 \%$

Proporción de positividad de controles $46 / 46+74=38 \%$

Razón de disparidad (Rd) $10 \times 74 / 50 \times 41=0.32$

FRECUENCIA SIMULTANEA DE INFECCION ENDOCERCIVAL Y ENDOMETRIAL POR CHLAMYDIA TRACHOMATIS

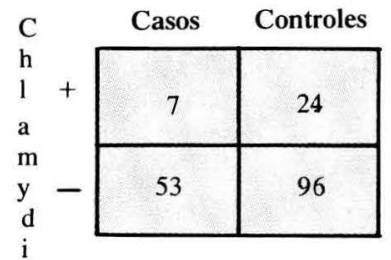

\author{
Proporción de positividad de casos \\ $7 / 7+53=12 \%$ \\ Proporción de positividad \\ de controles \\ $24 / 24+96=20 \%$ \\ Razón de disparidad (Rd) \\ $7 \times 96 / 52 \times 24=0.53$
}

endosalpinx y provocar en este último lesiones obstructivas, principalmente cuando la infección es a repetición o de larga duración, sería razonable postular que este gérmen tiene un papel muy importante en el origen de la infertilidad, principalmente a nivel tubo peritoneal. (32).

En nuestro estudio se encontró una mayor frecuencia de infección por $\mathrm{C}$. trachomatis en el grupo control que en el grupo de mujeres infértiles, pero sólo fue significativo en el endometrio del grupo control.

Estos resultados revelan una alta frecuencia de infección por Chlamydia en nuestro medio, no sólo en mujeres infértiles sino también en el grupo de mujeres fértiles, información que es coherente con los diferentes informes acerca de la frecuencia de infección clamidiásica masculina en nuestro medio; en un estudio hecho por Restrepo y colaboradores (33) se halló C. trachomatis en un $31 \%$ de hombres con uretritis; más recientemente, se ha hallado con frecuencia esta bacteria en pacientes que consultan por uretritis no gonocóccica (34); debido a que el hombre con clamidiasis es sintomático con mayor frecuencia que la mujer, y que en ésta la infección pasa casi desapercibida, es más probable en ellas la persistencia de la infección, la ausencia de trata- 
miento y por lo tanto, una mayor población de infectadas que se convierte en fuente de contagio; cabe entonces llamar la atención acerca de la importancia del tratamiento de las compañeras sexuales de todo hombre con uretritis no gonocóccica.

Tabla No. 3

FRECUENCIA DE INFECCION POR CHLAMYDIA TRACHOMATIS EN GRUPOS DE CASOS Y CONTROLES, EN ESTUDIOS EFECTUADOS EN ENDOCERVIX, ENDOMETRIO Y SIMULTANEO

\begin{tabular}{|l|lll|}
\hline \multirow{2}{*}{ No. Pacientes } & \multicolumn{3}{|c|}{ Muestra } \\
\cline { 2 - 4 } & Endocervix & Endometrio & Simultáneo \\
\hline Controles 120 & $39(32.5 \%)$ & $46(38 \%)$ & $24(20 \%)$ \\
Casos 60 & $12(20 \%)$ & $10(17 \%)$ & $7(12 \%)$ \\
$P$ & 0,11 & 0,005 & 0,23 \\
\hline
\end{tabular}

Para explicar la mayor frecuencia de infección por Chlamydia en el grupo control se requieren otras investigaciones; es concebible que diferencias socioeconómicas entre los casos y los controles de este estudio sean parte de la explicación; en efecto, es más bajo el nivel socioeconómico de las pacientes que acuden al H.U.S.V.P. que el de las que consultan a Profamilia y se sabe, por estudios en otros países, (24) que hay diferencias en las etiología de la uretritis masculina según el estrato socioeconómico y la raza.

Circunstancias de otra índole también podrían ser invocadas como hipótesis para explicar la diferencia pero reque- rirían estudios adicionales; por ejemplo: pudiera postularse que en la población de parejas fértiles es más probable la promiscuidad sexual de el esposo y, por ende, el riesgo de adquirir Chlamydia; en las parejas infértiles, por el contrario, la búsqueda del hijo puede ser un determinante de fidelidad conyugal y, como consecuencia de menor exposición al contagio.

Existen pocos estudios sobre la presencia de C. trachomatis en el endometrio (22); ellos tienen la dificultad obvia de la posible contaminación en el momento de obtener la muestra con material endocervical; por eso consideramos de importancia la implementación de esta técnica sencilla para la toma de muestas en la cavidad endometrial; su efectividad quedó corroborada al hallar positividad aislada de endometrio o de endocervix.

Nuestro estudio tiene la importancia, a diferencia de los publicados en la literatura consultada, que es el único que utiliza este modelo de investigación (Casos y Controles) y que analiza el mismo tiempo muestra endometrial y endocervical.

\section{Agradecimientos}

Al ICFES, a la Universidad de Antioquia y al Centro de Invetigaciones Médicas por su financiación y apoyo. Al Dr. Aníbal Castañeda L., Director de Profamilia; al Dr. Luis Fernando Isaza, Médico, Profamilia. A la bacterióloga María Victoria Gómez R., Laboratorio Departamental, S.S.S.A.; al señor Heriberto Henao, estadígrafo del CIM, a las Bacteriólogas Beatriz Eugenia Restrepo C. y Gilma Pineda, a la enfermera auxiliar Amanda Olaya, por su valiosa colaboración en la recolección de datos y ejecución del proyecto.

\section{BIBLIOGRAFIA}

1. Sweet RL, Schachter J, Landers DV Chlamydial infections in Obstetrics and Gynecology. Clin Obstet Gynecol 1983; 26: 145-146.

2. Westrom L. Gynecological chlamydial infections. Infection 10 (Suppl) $1982 ; 40-45$

3. Kiviat NB et al Cytologic manifestations of cervical and vaginal infections. JAMA 1985; 253: 997-1000.

4. Wentworth BB et al. Isolation of viruses, bacteria and other organism from veneral disease clinic patients: Methodology and problems associated with multiple isolation. Health Lab. Sci. 1973; 10: 75-81.

5. Hobson D. et al. Simplified method for diagnostic of genital and ocular infections with Chlamydia. Lancet 1974; 2: 555-556.

6. Oriel JD. et al. Chlamydia infections of the cervix. Br. J. Vener Dis 1974; 50: 11-16.

7. Burns DCM. et al. Isolation of Chlamydia form women attending a clinic for sexually transmitted diseases. Br J Vener Dis 1975; 51: 314-318.

8. Schachter J. et al. Are Chlamydial infections the most prevalent venereal disease? JAMA 1975; 231: 1252-1255

9. Nayyar JC et al. Isolation of Chlamydia trachomatis from women attending a clinic from sexually transmitted diseases. $\mathrm{Br} \mathbf{J}$ Vener Dis 1976; 52: 396-398

10. Ridgway GL, Oriel JD. Interrelationships of Chlamydia trachomatis and other pothogens in the female genital tract J Clin Pathol 1977; 30: 933-936.

11. Rees $\mathbf{E}$. et al. Chlamydia in relation to cervical infection and pelvic inflammatory disease In: Nongonococcal urethritis and related infections Ed. D. Hobson and K.K. Holmes American Society for Microbiology, Washington D.C., 1977.

12. Woolfitt JMG. Watt L. Chlamydial infection of the urogenital tract in promiscuous and non-promiscuous women. Br J Vener Dis 1977; 53: 93-95

13. Hughes EG, Mowatt J, Spence J. Endocervical Chlamydia trachomatis infection in Canadian adolescents. CMAJ 1989; 140: 297-301.

14. Saltz GR, Linnemann CC, Brookman RR, Rauh JL. Chlamydia trachomatis cervical infections in female adolescents. J. Pediatr 1981;98: 981 .

15. Eagar RM, Beach RK, Davidson AJ, et al. Epidemiologic and clinical factors of Chlamydia trachomatis in black, hispanic and white female adolescents. West J Med 1985; 143: 37-41.

16. Toomey KE, Rafferty MP, Stamm WE. Unrecognized high prevalence of Chlamydia trachomatis cervical infection in an isolated alaskan eskimo population. JAMA 1987; 258: 53-56.

17. Sánchez, RM y col. Detección de Infección endocervical por clamidia comparando la tinción de Papanicolaou inmunofluorescencia directa. Ginec Obst. Mex. 1989; 57: 29-36.

18. Schachter J. Stoner E, Moncada J. Screening for Chlamydial infections in women attending family planning clinics. West J Med 1983; 138: $375-379$.

19. Gjoannaess H., Knut D., Anestad G. et al. Pelvic inflammatory disease: etiologic studies with emphasis on Chalamydial infection. Obstet Gynecol 1982; 59: 550-555.

20. Moller BR, Ahrons S, Laurin J. Mardh P-A. Pelvic infection after elective abortion associated with Chlamydia trachomatis. Obstet Gynecol. 1982; 59: 210-213.

21. Shiootz H, Csángó PA. A prospective study of Chlamydia trachomatis in firts trimester abortion. Ann Clinic Res. 1985; 17: 60-63.

22. Sweer RL, Schachter J, Robbie MO. Failure of B-Lactam Antibiotics to eradicate Chlamydia trachomatis in the endometrium despite apparent clinical cure of acute salpingitis. JAMA 1983; 250: 2641-2645. 
23. Mardh P-A, Ripa KT, Svensson L, Westrom L. Chlamydia trachomatis in patients with acute salpingitis. N Engl J Med 1977;296: 1377.

24. Aral, SO, Holmes K.K. Epidemiology of sexually transmitted diseases. In: Holmes K.K. Mardh, PA, Sparling. P.F., Wiesner P.J. Eds: Sexually transmitted diseases, McGraw-Hill Books Co. New York, 1984; pp. 127-141.

25. Gump DW, Dickstein S, Gibson M. Endometritis related to Chlamy. dia trachomatis infection. Ann Intern Medic 1981; 95: 61-63.

26. Schachter J. Isolation of bedsoniae from human arthristis and abortion tissues. Am J Ophthalmol. 1967; 63: 1082.

27. Cracea E, Botez D. Genital mycoplasma and Chlamydiae in infertility and abortion. Arch. Reum Pathol Exp Microbiol 1981; 40: 107.

28. Tho PT, Byrd JR, McDonough PG Etiologies and subsequent reproductive performance of 100 couples with recurrent abortion. Fertil. Steril. 1979; 32: 389-395.

29. Pedersen BS, S. Stray-Pedersen. Etiologiec factors and subsequent reproductive performance in 195 couples with a prior history of habitual abortion. Am J Obstet Gynecol 1984; 148(2): 140.

30. Syva-Microtrak. Chlamydia trachomatis Direct specimen test. Syva Company.

31. Storz J, W. Tood, L. Schnorr. Chlamydial Infections: Breach of Host cellular Barriers. En: Virulence Mechanisms of Bacterial Pathogens. J.A. Roth. Eds. American Society for Microbiology. Wash. D.C. 1988 pp 161-181.

32. Stamm, W.E., Holmes, K.K. Chlamydia trachomatis infections of the adult. In: Holmes K.K., Mardh, Pa, Sparling P.F., Wiesner, P,J. Eds: Sexually Transmitted diseases, McGraw-Hill Books Co. New York, 1984 pp. 259-269.

33. Restrepo, M., Díaz F., Gómez M. Uretritis Masculina. Importancia de la Chlamydia trachomatis y de otros agentes. Acta Médic. Colombiana 1986; 11(1): 21-27.

34. Díaz F., Comunicación personal. 